\section{UJMM

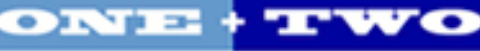

Volume 2 | 2010 Spring

\section{Undergraduate Journal of Mathematical}

Modeling: One + Two

2010

\title{
Quantum Mechanical Example of Anti-Tunneling
}

Tim Mitchell

University of South Florida

\author{
Advisors: \\ Masahiko Saito, Mathematics and Statistics \\ Scott Campbell, Chemical \& Biomedical Engineering \\ Problem Suggested By: Scott Campbell
}

Follow this and additional works at: https://digitalcommons.usf.edu/ujmm

Part of the Mathematics Commons

UJMM is an open access journal, free to authors and readers, and relies on your support: Donate Now

\section{Recommended Citation}

Mitchell, Tim (2010) "Quantum Mechanical Example of Anti-Tunneling," Undergraduate Journal of Mathematical Modeling: One + Two: Vol. 2: Iss. 2, Article 6.

DOI: http://dx.doi.org/10.5038/2326-3652.2.2.6

Available at: https://digitalcommons.usf.edu/ujmm/vol2/iss2/6 


\title{
Quantum Mechanical Example of Anti-Tunneling
}

\author{
Abstract \\ The probability of a particle being reflected by a semi-infinite energy barrier was solved using \\ Schrödinger's equations that describe the quantum mechanical behavior of the particle. The continuity of \\ the functions at the origin allowed the wave functions and their respective derivatives to be equated. \\ Solution of the resulting system yielded values $A_{1}$ and $B_{1}$ which were used to calculate the reflection \\ coefficient, $\left(B_{1} / A_{1}\right)^{2}$. The conclusions obtained from these results stated that the particle would be \\ reflected by the barrier when $\mathrm{E}$ \\ Keywords \\ Quantam Anti Tunneling, Schrodinger's Equation, Reflection Coefficient \\ Creative Commons License \\ (c) $($ ) $\Theta \Theta$
}

This work is licensed under a Creative Commons Attribution-Noncommercial-Share Alike 4.0 License. 


\section{TABLE OF CONTENTS}

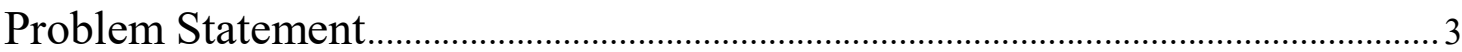

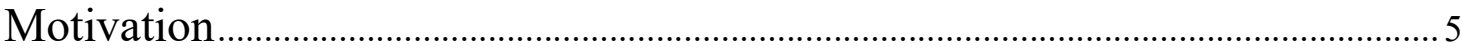

Mathematical Description and Solution Approach …..............................................

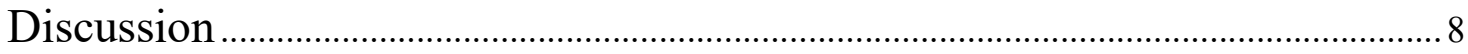

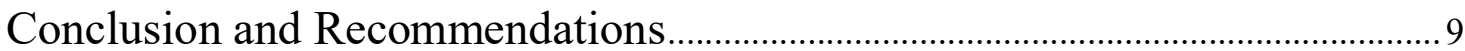

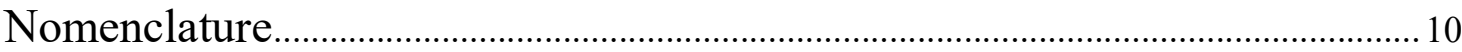

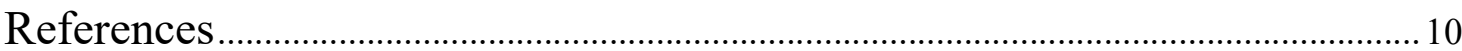

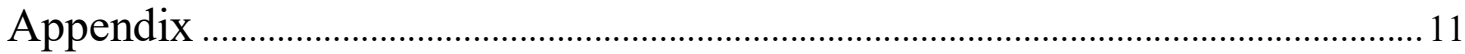




\section{PROBLEM STATEMENT}

As suggested by Scott Campbell

The model shown below describes a situation in which a particle of energy $(E)$ is "shot" at an energy barrier (infinite in the positive $x$ direction) with potential $(V)$. If the reflection coefficient is defined to be the probability that the particle will be reflected by the barrier, the classical reflection coefficient would look like that shown in the right hand figure - any particle with energy less than $V$ will be reflected and any with energy greater than $V$ will pass into the barrier. For very small particles, quantum mechanics, rather than classical mechanics, is the accurate description of the particle's behavior - and it yields a different result.
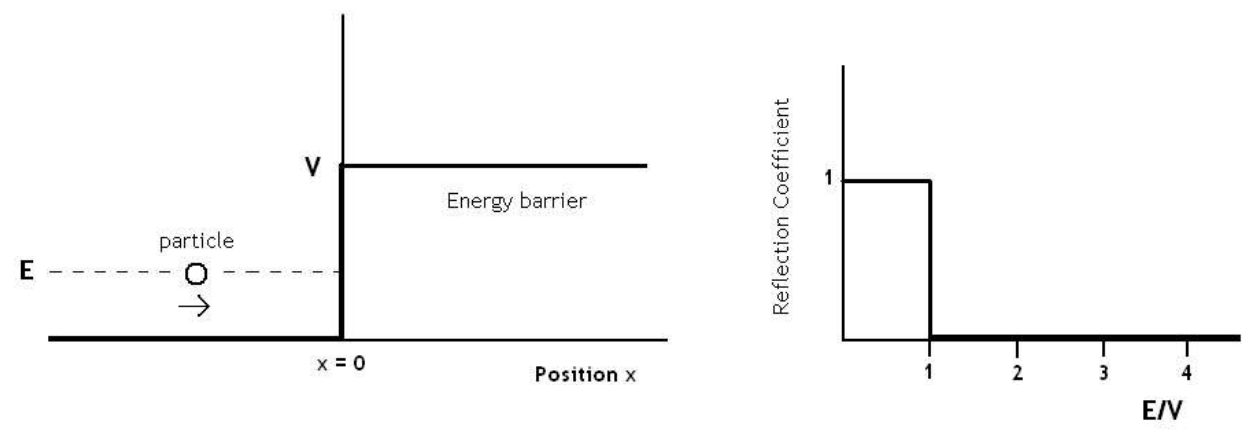

For the situation shown above, the quantum mechanical description of the particle's behavior is given by the following forms of the Schrödinger equation:

$$
\begin{array}{ll}
\frac{h^{2}}{8 \pi^{2} m} \frac{d^{2} \Psi_{1}}{d x^{2}}=-E \Psi_{1} & x<0 \\
\frac{h^{2}}{8 \pi^{2} m} \frac{d^{2} \Psi_{2}}{d x^{2}}=-(E-V) \Psi_{2} & x \geq 0
\end{array}
$$

where $\Psi_{1}$ and $\Psi_{2}$ are the wave functions for the particle for $x<0$ and $x \geq 0$, respectively, $E$ is the particle energy, $m$ is the particle mass, $V$ is the potential height of the barrier and $h$ is Planck's constant. 
The goal of this project was to evaluate the reflection coefficient for the quantum mechanical description (for the case of $E>V$ ).

The first objective was to prove that, for suitable choices of $r$ and $k$,

$$
\Psi_{1}=A_{1} e^{i r x}+B_{1} e^{-i r x} \quad \Psi_{2}=A_{2} e^{i k x}+B_{2} e^{-i k x}
$$

were solutions to the Schrödinger equations (1) and (2) where $A_{1}, B_{1}, A_{2}$ and $B_{2}$ are unknown constants. Also, explicit expressions for $r$ and $k$ were found.

The second objective was to evaluate the reflection coefficient, defined as

$$
\frac{\left|B_{1}\right|^{2}}{\left|A_{1}\right|^{2}}
$$

In the above wave equations for $\Psi_{1}$ and $\Psi_{2}$, the terms $e^{i r x}$ and $e^{i k x}$ represent a particle moving to the right (with respect to the $x$ direction) while $e^{-i r x}$ and $e^{-i k x}$ represent a particle moving to the left (with respect to the $x$ direction). A particle inside the barrier will move only to the right but a particle to the left of the barrier may move either to the right (if "shot") or left (if reflected). The wave function $\Psi$ and its first derivative were assumed to be continuous at $x=0$.

Finally, the reflection coefficient as a function of $E / V$ (for values of $E / V \geq 1$ ) was plotted and appears in the Appendix. 


\section{MOTIVATION}

The quantum mechanical example of anti-tunneling is useful because it shows that subatomic and atomic particles behave differently from the classical mechanics model. Antitunneling shows that a particle possesses some chance of being reflected from a barrier; provided that barrier has less energy than the particle. This is analogous, in classical mechanics, to throwing a baseball with a great force at a window and having the baseball reflect off of it. The objective of this project was to find the probability of a particle being reflected from a barrier.

\section{MATHEMATICAL DESCRIPTION AND SOLUTION APPROACH}

The following two equations describe the motion of the wave:

$$
\begin{array}{ll}
\frac{\mathrm{h}^{2}}{8 \pi^{2} \mathrm{~m}} \frac{\mathrm{d}^{2} \Psi_{1}}{\mathrm{dx^{2 }}}=-E \Psi_{1} & \mathrm{x}<0, \\
\frac{\mathrm{h}^{2}}{8 \pi^{2} \mathrm{~m}} \frac{\mathrm{d}^{2} \Psi_{2}}{\mathrm{dx^{2 }}}=-(E-V) \Psi_{2} & \mathrm{x} \geq 0
\end{array}
$$

where $\Psi_{1}$, and $\Psi_{2}$ are the wave functions of the particle introduced in the problem statement.

The solution of these equations is given by

$$
\begin{aligned}
& \Psi_{1}=A_{1} e^{i r x}+B_{1} e^{-i r x} \\
& \Psi_{2}=A_{2} e^{i k x}+B_{2} e^{-i k x} .
\end{aligned}
$$

Evaluating the first and second derivatives of $\Psi_{1}$ yields

$$
\begin{aligned}
& \frac{d \Psi_{1}}{d x}=i r A_{1} e^{i r x}-i r B_{1} e^{-i r x} \\
& \frac{d^{2} \Psi_{1}}{d x^{2}}=-r^{2} A_{1} e^{i r x}-r^{2} B_{1} e^{-i r x}
\end{aligned}
$$

and evaluating the first and second derivatives of $\Psi_{2}$ gives 


$$
\begin{aligned}
& \frac{d \Psi_{2}}{d x}=i k A_{2} e^{i k x}-i k B_{2} e^{-i k x} \\
& \frac{d^{2} \Psi_{2}}{d x^{2}}=-k^{2} A_{2} e^{i k x}-k^{2} B_{2} e^{-i k x}
\end{aligned}
$$

Substitution of (3) and (6) into equation (1) gives

$$
\left(\frac{\hbar^{2}}{2 m}\right)\left(-r^{2} A_{1} e^{i r x}-r^{2} B_{1} e^{-i r x}\right)=-E\left(A_{1} e^{i r x}+B_{1} e^{-i r x}\right)
$$

where $\hbar=h / 2 \pi$. Solving for $\mathrm{r}^{2}$ yields

$$
r^{2}=\frac{2 m E}{\hbar^{2}} \frac{\left(A_{1} e^{i r x}+B_{1} e^{-i r x}\right)}{\left(A_{1} e^{i r x}+B_{1} e^{-i r x}\right)}=\frac{2 m E}{\hbar^{2}}
$$

which implies that

$$
r=\sqrt{\frac{2 m E}{\hbar^{2}}}
$$

Similarly, substituting (4) and (8) into equation (2) and once again letting $\hbar=h / 2 \pi$ gives

$$
\left(\frac{\hbar^{2}}{2 m}\right)\left(-k^{2} A_{2} e^{i k x}-k^{2} B_{2} e^{-i k x}\right)=-(E-V)\left(A_{2} e^{i k x}+B_{2} e^{-i k x}\right)
$$

and solving for $k^{2}$ results in

$$
k^{2}=\frac{2 m(E-V)}{\hbar^{2}} \frac{\left(A_{2} e^{i k x}+B_{2} e^{-i k x}\right)}{\left(A_{2} e^{i k x}+B_{2} e^{-i k x}\right)}=\frac{2 m(E-V)}{\hbar^{2}}
$$

i.e.

$$
\mathrm{k}=\sqrt{\frac{2 \mathrm{~m}(\mathrm{E}-\mathrm{V})}{\hbar^{2}}}
$$


Since each $\Psi(\mathrm{x})$ and $\frac{\mathrm{d} \Psi}{\mathrm{dx}}$ are continuous at $x=0,(3)$ and (4) says that

$$
\mathrm{A}_{1}+\mathrm{B}_{1}=\Psi_{1}(0)=\Psi_{2}(0)=\mathrm{A}_{2}+\mathrm{B}_{2}
$$

and (5) and (6) says that

$$
\mathrm{irA}_{1}-\operatorname{irB}_{1}=\frac{\mathrm{d} \Psi_{1}}{\mathrm{dx}}(0)=\frac{\mathrm{d} \Psi_{2}}{\mathrm{dx}}(0)=\mathrm{ikA} \mathrm{A}_{2}-\mathrm{ikB} \mathrm{B}_{2}
$$

or simply that

$$
A_{1}-B_{1}=\frac{k}{r}\left(A_{2}-B_{2}\right)
$$

However, $\mathrm{B}_{2}=0$ since the particle is not traveling towards the left-hand $x$-direction after entering the barrier. Therefore, substituting $B_{2}=0$ into equations (11) and (12) yields,

$$
\mathrm{A}_{1}-\mathrm{B}_{1}=\frac{\mathrm{k}}{\mathrm{r}} \mathrm{A}_{2} \quad \text { and } \quad \mathrm{A}_{1}+\mathrm{B}_{1}=\mathrm{A}_{2}
$$

and solving this system of equations yields

$$
\begin{aligned}
& \mathrm{A}_{1}=\frac{1}{2} \mathrm{~A}_{2}\left(1+\frac{\mathrm{k}}{\mathrm{r}}\right) \\
& \mathrm{B}_{1}=\frac{1}{2} \mathrm{~A}_{2}\left(1-\frac{\mathrm{k}}{\mathrm{r}}\right)
\end{aligned}
$$

Simplifying the expression of $\frac{\mathrm{k}}{\mathrm{r}} \mathrm{using}(9)$ and (10) gives

$$
\frac{\mathrm{k}}{\mathrm{r}}=\frac{\sqrt{\frac{2 \mathrm{~m}(\mathrm{E}-\mathrm{V})}{\hbar^{2}}}}{\sqrt{\frac{2 \mathrm{mE}}{\hbar^{2}}}}=\sqrt{\frac{2 m \hbar^{2}(E-V)}{2 m \hbar^{2} E}}=\sqrt{\frac{E-V}{E}}=\sqrt{1-\frac{V}{E}}=\sqrt{1-\frac{1}{E / V}}
$$

The reflection coefficient is defined as $\left(\frac{B_{1}}{A_{1}}\right)^{2}$. Equations (13), (14) and (5) imply that 


$$
\left(\frac{B_{1}}{A_{1}}\right)^{2}=\left(\frac{\frac{1}{2} A_{2}\left(1-\frac{k}{r}\right)}{\frac{1}{2} A_{2}\left(1+\frac{k}{r}\right)}\right)^{2}=\left(\frac{\left(1-\frac{k}{r}\right)}{\left(1+\frac{k}{r}\right)}\right)^{2}=\left(\frac{\left(1-\sqrt{1-\frac{1}{E / V}}\right)}{\left(1+\sqrt{1-\frac{1}{E}}\right)}\right)^{2}
$$

A graph of the reflection coefficient vs. $E / V$ appears in the Appendix.

\section{DISCUSSION}

The equation for the reflection coefficient (16) describes the probability of the particle being reflected from the barrier. The graph (see Appendix) helps to visualize how the chance of reflection related to $E / V$. As shown, the particle has a very high chance of being reflected when $E / V \approx 1$. This can also be explained by considering the limit of the function as $E / V$ approaches one.

$$
\lim _{\overline{\mathrm{V}} \rightarrow 1}\left(\frac{\left(1-\sqrt{1-\frac{1}{\mathrm{E} / \mathrm{V}}}\right)}{\left(1+\sqrt{1-\frac{1}{\mathrm{E} / \mathrm{V}}}\right)}\right)^{2}=1
$$

However, as the energy of the particle increases, the probability of the particle being reflected decreases at an exponential rate. This probability approaches 0 as $\mathrm{E} / \mathrm{V}$ increases:

$$
\lim _{\overrightarrow{\mathrm{V}} \rightarrow \infty}\left(\frac{\left(1-\sqrt{1-\frac{1}{\mathrm{E} / \mathrm{V}}}\right)}{\left(1+\sqrt{1-\frac{1}{\mathrm{E} / \mathrm{V}}}\right)}\right)^{2}=0
$$

This result is as expected. If the limit did not converge, there would be an unreasonably high probability that particles will be reflected off of the energy barrier. 


\section{CONCLUSION AND RECOMMENDATIONS}

After graphing the reflection coefficient of the particle (see Appendix), counterintuitive results were obtained. Even though the particle had more than enough energy to penetrate the barrier, it still had some small chance of being reflected. This discovery suggested that subatomic particles do not act in a manner described by classical physics and new models are required to describe their behaviors. The summary of this graph showed that the probability of the particle being reflected decreases exponentially as the ratio of $E / V$ increases. One possible extension of this project would be to solve for the reflection coefficient considering the case where $V>E$. 


\title{
NOMENCLATURE
}

\author{
$\mathrm{h}=$ Planck's constant $=6.626 * 10^{-34}\left(\frac{\mathrm{m}^{2} \mathrm{~kg}}{\mathrm{~s}}\right)$ \\ $\hbar=\frac{h}{2 \pi}\left(\frac{m^{2} \mathrm{~kg}}{\mathrm{~s}}\right)$ \\ $\mathrm{m}=$ the particle mass $(\mathrm{kg})$ \\ $E=$ the particle energy $(J)$ \\ $\mathrm{V}=$ the potential height of the barrier $(\mathrm{J})$ \\ $\Psi=$ the wave function for the particle
}

\section{REFERENCE}

Ferry, D. "Quantum Mechanics, An Introduction for Device Physicists and Electrical Engineers. Second Edition.” Taylor \& Francis Group, New York. (2001) 


\section{APPENDIX}

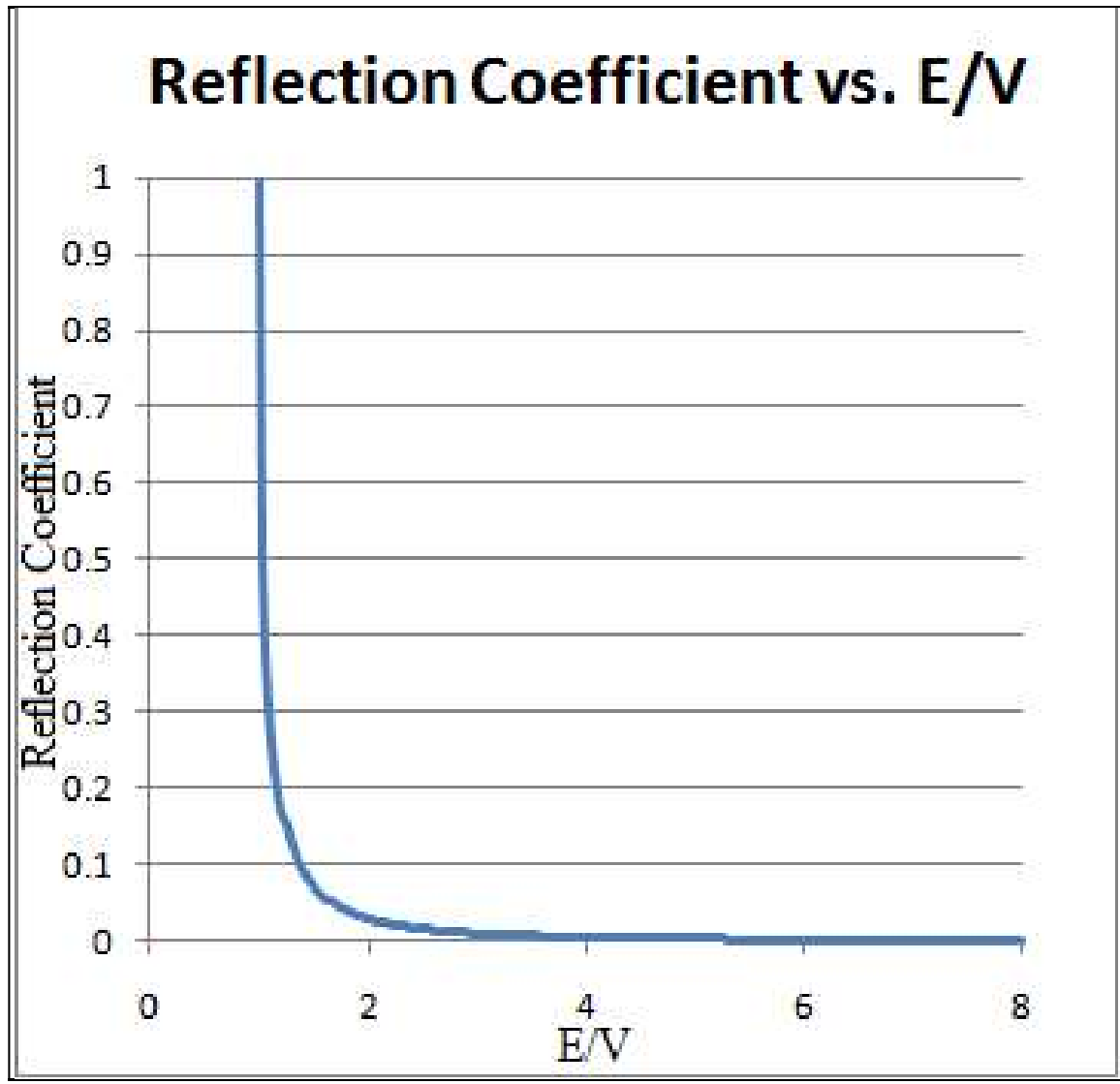

